\title{
Neural Activity Protects Hypothalamic Magnocellular Neurons against Axotomy-Induced Programmed Cell Death
}

\author{
Tal Shahar, ${ }^{*}$ Shirley B. House, ${ }^{\star}$ and Harold Gainer \\ Laboratory of Neurochemistry, National Institute of Neurological Disorders and Stroke, National Institutes of Health, Bethesda, Maryland 20892
}

\begin{abstract}
Axotomy typically leads to retrograde neuronal degeneration in the CNS. Studies in the hypothalamo-neurohypophysial system (HNS) have suggested that neural activity is supportive of magnocellular neuronal (MCN) survival after axotomy. In this study, we directly test this hypothesis by inhibiting neural activity in the HNS, both in vivo and in vitro, by the use of tetrodotoxin (TTX). After median eminence compression to produce axonal injury, unilateral superfusion of $3 \mu \mathrm{M}$ TTX into the rat supraoptic nucleus (SON), delivered with the use of a miniature osmotic pump for 2 weeks in vivo, produced a decrease in the number of surviving MCNs in the TTX-treated SON, compared with the contralateral untreated side of the SON. In vitro application of $2.5 \mu \mathrm{M}$ TTX for 2 weeks to the SON in organotypic culture produced a 73\% decrease in the surviving MCNs, compared with untreated control cultures. Raising the extracellular $\mathrm{KCl}$ in the culture medium to $25 \mathrm{~mm}$ rescued the MCNs from the axotomy- and TTX-induced cell death. These data support the proposal that after axotomy, neural activity is neuroprotective in the HNS.
\end{abstract}

Key words: hypothalamus; vasopressin; oxytocin; magnocellular neuron; axotomy; apoptosis

\section{Introduction}

It is well known that axotomy leads to neuronal degeneration in the CNS (Ramon y Cajal, 1928), and the vasopressin (VP) and oxytocin (OT) magnocellular neurons (MCNs) of the hypothalamo-neurohypophysal system (HNS) have served as classical in vivo models for the study of this phenomenon. Various forms of axonal injury such as hypophysectomy (Hare, 1937; Raisman, 1973), neuro-hypophysectomy (Bodian and Maran, 1951), pituitary stalk compression (Dohanics et al., 1992), and pituitary stalk transection (Alonso et al., 1996) have been shown to lead to retrograde neurodegeneration of $>75 \%$ of the MCNs. The axons distal to the transection site degenerate and are eliminated by activated microglia and pituicytes (Dellmann, 1973; Mander and Morris, 1994). The axons of the surviving MCNs can regenerate by sprouting into the external layer of the median eminence (ME) (Moll, 1962; Raisman, 1973; Kawamoto and Kawashima, 1985), the proximal edge of the transected stalk (Moll, 1962; Daniel and Prichard, 1975), and the third ventricle (Wu et al., 1989; Scott et al., 1995) to form alternative neurohemal organs (Hare, 1937; Raisman, 1973) that can eventually alleviate the diabetes insipidus produced by the lesion (O'Connor, 1952;

Received March 10, 2004; revised May 20, 2004; accepted June 9, 2004.

We thank Dr. Riccardo Cassiani-Ingoni for his graphical rendering of the lesioning device described in this study and Dr. Elka Scordalakes for her careful reading of this manuscript.

*T.S. and S.B.H. contributed equally to this work. T.S. contributed predominately to the in vivo experiments, and S.B.H. contributed predominately to the in vitro experiments.

Correspondence should be addressed to Dr. Harold Gainer, National Institute of Neurological Disorders and Stroke, National Institutes of Health, Building 36, Room 4D-04, Bethesda, MD 20892-4130. E-mail: gainerh@ninds.nih.gov.

T. Shahar's present address: The Tel Aviv Sourasky Medical Center, 6 Weizmann Street, Tel Aviv, 64239, Israel. D01:10.1523/JNEUROSCI.0886-04.2004

Copyright $\odot 2004$ Society for Neuroscience $\quad$ 0270-6474/04/246553-10\$15.00/0
Moll, 1962; Dellmann, 1973; Raisman, 1973; Daniel and Prichard, 1975; Dellmann et al., 1988).

Treatments designed to counter the acute diabetes insipidus caused by axotomy in the HNS, such as the administration of exogenous vasopressin, lead to a greater retrograde degeneration of the MCNs (Herman et al., 1986, 1987). Chronic hyponatremia, which inhibits secretory activity in the MCNs (Verbalis et al., 1986; Robinson et al., 1990; Verbalis and Dohanics, 1991), also produces an enhanced degeneration of the MCNs after axonal injury in the HNS (Dohanics et al., 1996). Because both of these treatments lead to decreased functional activity of the MCNs, it was suggested that there is a neuroprotective role of functional activity in the HNS after axonal injury (Herman et al., 1987; Dohanics et al., 1996). The latter view is also consistent with the findings of Huang and Dellmann (1996), who showed that chronic intermittent salt loading, which increases the functional activity of the MCNs in the HNS, produced an increased survival of the VP MCNs after hypothalamic stalk transection.

In this study, we directly test the hypothesis that functional activity of the MCNs plays a neuroprotective role in the HNS after axonal injury by using novel in vivo and in vitro experimental models. We found both in the in vivo and in vitro studies that inhibition of neural activity in the supraoptic nucleus (SON) by tetrodotoxin (TTX) significantly increased the extent of MCN degeneration after axotomy. In addition, we found that potassium depolarization rescues OT and VP MCNs from axotomyinduced neurodegeneration in vitro and also reverses the deleterious effect of TTX on the survival of both OT and VP MCNs in the SON.

\section{Materials and Methods}

Animals: in vivo experiments. All procedures were performed in accordance with the National Institutes of Health guidelines on the care and 
use of animal study protocol approved by the National Institute of Neurological Disorders and Stroke Animal Care and Use Committee. Adult male Sprague Dawley rats (250-350 gm body weight) (Taconic, Germantown, NY) were used. The animals were kept under standard laboratory conditions (room temperature, $22 \pm 1^{\circ} \mathrm{C} ; 12 \mathrm{hr}$ light/dark cycle, with lights on at 6:00 A.M.). Regular rat chow and water were provided ad libitum.

Neurosurgical interventions. Rats were weighed and anesthetized with Isoflurane, using a Stoelting (Kiel, WI) gas anesthesia adaptor for the stereotaxic instrument. Once anesthetized, the rat was placed in the stereotaxic frame (David Kopf Instruments, Tujunga, CA), the incision site was prepared with alternating scrubs of betadine and alcohol, and a $2 \mathrm{~cm}$ midsagittal skin incision was made on the rat calvaria. Using the stereotaxic coordinates from the Paxinos and Watson (1986) atlas, two types of experimental surgical procedures were performed.

ME axonal compression. A small (2.5 mm width, $1.5 \mathrm{~mm}$ thick) midsagittal burr hole, located $3.2 \mathrm{~mm}$ posterior to the bregma, was made on the skull using a dental burr (see Fig. 1A). An L-shaped wire held in a device specially designed to apply constant pressure (see Fig. $1 B$ ) was lowered into the brain at the level of the ME to compress the neurohypophysial axons. The stereotaxic coordinates used were $3.2 \mathrm{~mm}$ posterior to the bregma, $0 \mathrm{~mm}$ mediolateral. The L-shaped wire was lowered until it met the resistance of the skull base, and the compression was maintained for $5 \mathrm{~min}$. Before the descent of the L-shaped wire, the superior sagittal sinus was carefully deviated laterally to prevent its rupture and bleeding.

All of the experimental surgeries were histologically confirmed by visual inspection of the location and size of the ME compression damage. After perfusion, $30 \mu \mathrm{m}$ coronal sections were cut from the compressed area and stained by Giemsa stain (Sigma-Aldrich, St. Louis, MO) to locate the characteristic $2 \mathrm{~mm}$ lesion at the level of the ME at the base of the brain. Rats with incomplete compression (i.e., deviating from the midline or having an inadequate extent of ME damage) were excluded from the study. In addition, we also monitored the rats for symptoms of diabetes insipidus as a functional criterion for effective damage of the HNS axons.

Placements of intracerebral cannulas and osmotic pump installation. A small ( $1.5 \mathrm{~mm}$ diameter) parasagittal hole was made on each side of the skull $(1.8 \mathrm{~mm}$ lateral to the sagittal suture and $1.30 \mathrm{~mm}$ caudal to the bregma) using dental burr (Fig. 1C). Two sterile stainless-steel cannulas (28 gauge), $8.80 \mathrm{~mm}$ long, were inserted down to a level of $0.3 \mathrm{~mm}$ up to the dorsal border of the SON using the following stereotaxic coordinates: $1.30 \mathrm{~mm}$ posterior to the bregma; $1.80 \mathrm{~mm}$ mediolateral on each side; $8.80 \mathrm{~mm}$ dorsoventral. Each cannula (Plastics One, Roanoke, VA) was attached via sterile polyethylene (PE) 100 tubing (Becton Dickinson, Sparks, MD) to a sterile model 2002, Alzet (Cupertino, CA) miniature osmotic pump and fixed to the skull with sterile acrylic dental cement (Plastics One) bonded to sterile stainless-steel screws (Plastics One) inserted in the skull. The two Alzet miniature osmotic pumps, connected to the PE100 tubes, were placed subcutaneously on the dorsal aspect of the rat, between the two scapulas through subcutaneous tunnels, which were formed using hemostatic forceps starting from the midsagittal skin incision. One pump (for the left SON) contained the vehicle only (sterile $\mathrm{PBS}$ ), whereas the other pump (for the right SON) contained $3 \mu \mathrm{M}$ TTX in PBS filtered via a $0.22 \mu \mathrm{M}$ Millipore (Bedford, MA) filter. The pumps were filled at least $12 \mathrm{hr}$ before the surgery in a sterile environment and placed in the incubator $\left(37^{\circ} \mathrm{C}, 5 \% \mathrm{CO}_{2}\right)$ while immersed in PBS for the purpose of osmotic activation.

After the above neurosurgical interventions, the sagittal skin incision was closed with surgical stainless-steel clips, and Ketoprofen was administered $(5 \mathrm{mg} / \mathrm{kg}$, diluted in $0.9 \% \mathrm{NaCl})$. After surgery, the animals were maintained for $14 \mathrm{~d}$, after which they were anesthetized with pentobarbital sodium $(50 \mathrm{mg} / \mathrm{kg}$, i.p., body weight) and immediately perfused transcardially with ice-cold $50 \mathrm{ml}$ of PBS followed by $250 \mathrm{ml}$ of fixative solution (4\% paraformaldehyde, $0.19 \%$ picric acid in $0.1 \mathrm{M}$ phosphate buffer, $\mathrm{pH}$ 7.4). Brains were removed, postfixed overnight in the same fixative solution (diluted 1:1 with PBS), and further processed for fluorescence immunohistochemistry as described below.

Immunohistochemistry for the in vivo experiments. After the perfusion and overnight postfixation at $4^{\circ} \mathrm{C}$, we placed the brains in $30 \%$ sucrose for $24 \mathrm{hr}$ at $4^{\circ} \mathrm{C}$ for cryoprotection. Serial coronal sections, $12 \mu \mathrm{m}$ thick, were cut through the SON region with a cryostat (Reichert-Jung 2800; Frigocut, Heidelberg, Germany) at $-18^{\circ} \mathrm{C}$ and placed on poly-L-lysinecoated glass slides. Each hypothalamic block containing the SON was cut to produce four parallel series, each containing five slides with six coronal sections per slide and with the same rostral to caudal topographic presentation of the SON. All mounted tissue sections were rinsed three times for $10 \mathrm{~min}$ in PBS and then blocked in 10\% normal goat serum (NGS) and $0.3 \%$ Triton X-100 in PBS for $1 \mathrm{hr}$ at room temperature followed by incubation in primary antibody in $5 \%$ bovine serum albumin in PBS. For the detection of Fos protein, we incubated the sections in a Fos antiserum (Oncogene Science, Uniondale, NY) diluted to 1:1000 for $24 \mathrm{hr}$ at $4^{\circ} \mathrm{C}$. To identify VP or OT neurons in the double-label experiments with Fos immunostaining, the mouse monoclonal antibody PS41, against VPneurophysin (NP), or the PS38 mouse monoclonal antibody against OTNP, respectively (Ben-Barak et al., 1985; Whitnall et al., 1985), were used at dilutions of 1:25 and were incubated overnight at $4^{\circ} \mathrm{C}$. After incubation in primary antibodies, the slides were rinsed three times for $10 \mathrm{~min}$ in PBS at room temperature and further incubated for $1 \mathrm{hr}$ at room temperature in Alexa 594-conjugated goat anti-rabbit (Molecular Probes, Eugene, OR) secondary antibody (1:1000 dilution in PBS) to visualize the Fos-IR as red fluorescent nuclei, and the VP- and OT-IR was detected by Alexa 488-conjugated goat anti-mouse (Molecular Probes) secondary antibody (1:1000 dilution in PBS) as cytoplasmic green fluorescence.

When double-label immunocytochemistry for VP-NP versus OT-NP was done, we used a polyclonal rabbit antibody, THR, against VP-NP (obtained from Dr. Allan Robinson, University of California Los Angeles School of Medicine, Los Angeles, CA) at a dilution of 1:2000, incubated overnight at $4^{\circ} \mathrm{C}$ and detected by Alexa 488 -conjugated goat anti-rabbit (Molecular Probes) secondary antibody (1:1000). To visualize the OT neurons, we used a mouse monoclonal antibody, PS38, against OT-NP (Ben-Barak et al., 1985; Whitnall et al., 1985) at a dilution of 1:25 and with overnight incubation at $4^{\circ} \mathrm{C}$. For the OT staining, the secondary antibody Alexa 594-conjugated goat anti-mouse second antibody (1:1000 dilution) was used. After immunohistochemistry, the sections were air dried overnight in the dark and coverslipped using Prolong Antifade Kit (Molecular Probes) mounting medium.

In addition to VP-NP and OT-NP immunohistochemical staining, all sections were stained with 4,6-diamidino-2-phenylindole (DAPI; SigmaAldrich), a cytochemical stain for DNA that labels cell nuclei. We counted only OT-NP or VP-NP MCNs that also contained DAPIpositive nuclei. We were not able to unequivocally identify DAPIpositive nuclei as MCNs, with their typically large round nuclei, without the simultaneous immunohistochemical staining for VP-NP or OT-NP.

Measurements of representative MCN numbers in the SON. VP-NP and OT-NP immunoreactive MCNs were counted in one section from each of five identifiable levels between the most rostral to the most caudal levels of the SON by using the Paxinos and Watson (1986) atlas as a guide. The first coronal section that was counted was at the most rostral region of the SON, corresponding to approximately $-0.8 \mathrm{~mm}$ from the bregma; the second section was adjacent to the most rostral level of the suprachiasmatic nucleus ( $\mathrm{SCN}$ ) corresponding to $-0.92 \mathrm{~mm}$ from the bregma and was identified by the positive staining of SCN neurons for VP-NP. The third section was at the level of the mid-SCN (approximately -1.3 $\mathrm{mm}$ ), where the characteristic tear shape of VP-NP-positive neurons in the SCN are found. The fourth section was at the most caudal level of the SCN (approximately $-1.4 \mathrm{~mm}$ ), and the fifth section was at the most caudal level of the SON (approximately $-1.7 \mathrm{~mm}$ ). The sums of the OT and VP MNCs from these five sections were compared between the experimental and control SONs.

Experiments: slice-explant organotypic cultures. Organotypic hypothalamic cultures were made as described previously (House et al., 1998; Rusnak et al., 2002). Briefly, 6-d-old Sprague Dawley pups (Taconic) were decapitated, and their brains were quickly removed. Hypothalamic tissue blocks were sectioned into $350 \mu \mathrm{m}$ coronal slices using a McIlwain tissue slicer (Stoelting, Wood Dale, IL). The four slices containing the entire extent of the SON were separated in $4^{\circ} \mathrm{C}$ Gey's Balanced Salt Solution (Invitrogen, Grand Island, NY) enriched with glucose ( $5 \mathrm{mg} / \mathrm{ml})$. 
Selected sections were trimmed and placed on Millicell-CM filter inserts (pore size, $0.4 \mu \mathrm{m}$; diameter, $30 \mathrm{~mm}$; Millipore). Each filter insert was placed in a Petri dish $(35 \mathrm{~mm})$ containing $1.2 \mathrm{ml}$ of culture medium. Incubation of the cultures was stationary in $5 \% \mathrm{CO}_{2}$ enriched air at $35^{\circ} \mathrm{C}$. Cultures were routinely maintained for $15 \mathrm{~d}$ in serum containing medium (SCM) with the presence of various pharmacological agents. Medium was replaced three times per week.

Media and incubations. The SCM was composed of 50\% Eagles Basal Medium with Earle's salts, 25\% heat inactivated horse serum, 25\% HBSS, $0.5 \%$ glucose, and $25 \mathrm{U} / \mathrm{ml}$ penicillin/streptomycin. The osmotic pressure of the standard medium was $314 \mathrm{mOsm} / \mathrm{l}$. In these experiments, slices cultured with and without various pharmacological agents were divided into experimental and control groups. The control groups underwent only a change of control media. Slices in both groups were fixed after $15 \mathrm{~d}$. The pharmacological agents used in the experimental groups were: TTX (2.5 $\mu \mathrm{m}$; Sigma), KCl (25 mm; Sigma), and ciliary neurotrophic factor (CNTF; $10 \mathrm{ng} / \mathrm{ml}$; Sigma).

Immunohistochemistry for in vitro experiments. After incubation in vitro for $15 \mathrm{~d}$, the explants on the filters were fixed in $4 \%$ formaldehyde in PBS for $1 \mathrm{hr}$, rinsed three times for $10 \mathrm{~min}$ in PBS, and then placed into cryoprotectant medium (Watson et al., 1986) and stored at $4^{\circ} \mathrm{C}$. For immunohistochemistry, the filters containing the fixed slices were excised from the inserts and placed in Netwell carriers (Costar, Cambridge, MA). Filters were rinsed in PBS and blocked in 10\% NGS containing $0.3 \%$ Triton X-100 for $2 \mathrm{hr}$. In some immunohistochemical procedures that used the PS 38, 41, and 45 mouse monoclonal antibodies, goat anti-mouse peroxidase-conjugated second antibodies were used, and the immunoreactivity was visualized as the diaminobenzidine reaction product (House et al., 1998). Double-label immunohistochemistry was also performed to distinguish the OT and VP MCNs in the SONs in the cultures. To identify the VP neurons, the polyclonal rabbit antibody (THR) against VP-neurophysin (obtained from Dr. Allan Robinson) was used at dilution of 1:2000 overnight at $4^{\circ} \mathrm{C}$ and was detected by Alexa 488-conjugated goat anti-rabbit (Molecular Probes) secondary antibody (1:500). To label OT neurons, a mouse monoclonal antibody against OT-neurophysin, PS 38 (Ben-Barak et al., 1985; Whitnall et al., 1985) was used at a dilution of 1:25 followed by Alexa 594-conjugated goat antimouse second antibody (1:500 dilution). After immunohistochemistry, the sections were eased off the filters using a number 0 or number 1 fine sable brush, placed on double-subbed slides, and allowed to dry overnight before counterstaining and mounting. Tissue that was too fragile to remove from the filters was counterstained in the netwells and mounted directly using Prolong antifade kit (Molecular Probes) mounting medium. Slides were viewed and cells were counted under an epifluorescence microscope (Nikon Labophot; Nikon, Tokyo, Japan) as described previously (House et al., 1998).

Statistical analyses. Statistical significance of differences between groups was calculated by one-way ANOVA followed by Fisher's protected least significant differences (PLSD) post hoc test. Results are expressed as mean \pm SEM, and differences between groups were considered statistically significant when $p$ values were $<0.05$.

\section{Results}

\section{The in vivo experimental system}

The goal of these in vivo studies was to determine whether the inhibition of the spontaneous electrical and synaptic activity in the SON by the local application of TTX would increase the axotomy-induced cell death of the MCNs. Figure 1 illustrates the approach that was used to produce axonal damage at the level of the ME by compression and to simultaneously superfuse the SON unilaterally with TTX for 2 weeks in vivo. We used ME compression (Dohanics et al., 1992, 1996) to cause axonal injury, because hypophysectomy or neurolobectomy, which use parapharyngeal approaches, are intrinsically more traumatic procedures. Other procedures such as ME or pituitary stalk transaction can lead to damage of the portal circulation and thereby compromise the blood supply to the anterior lobe and possibly produce hemorrhage at the base of the skull (Daniel and Prichard, 1975).
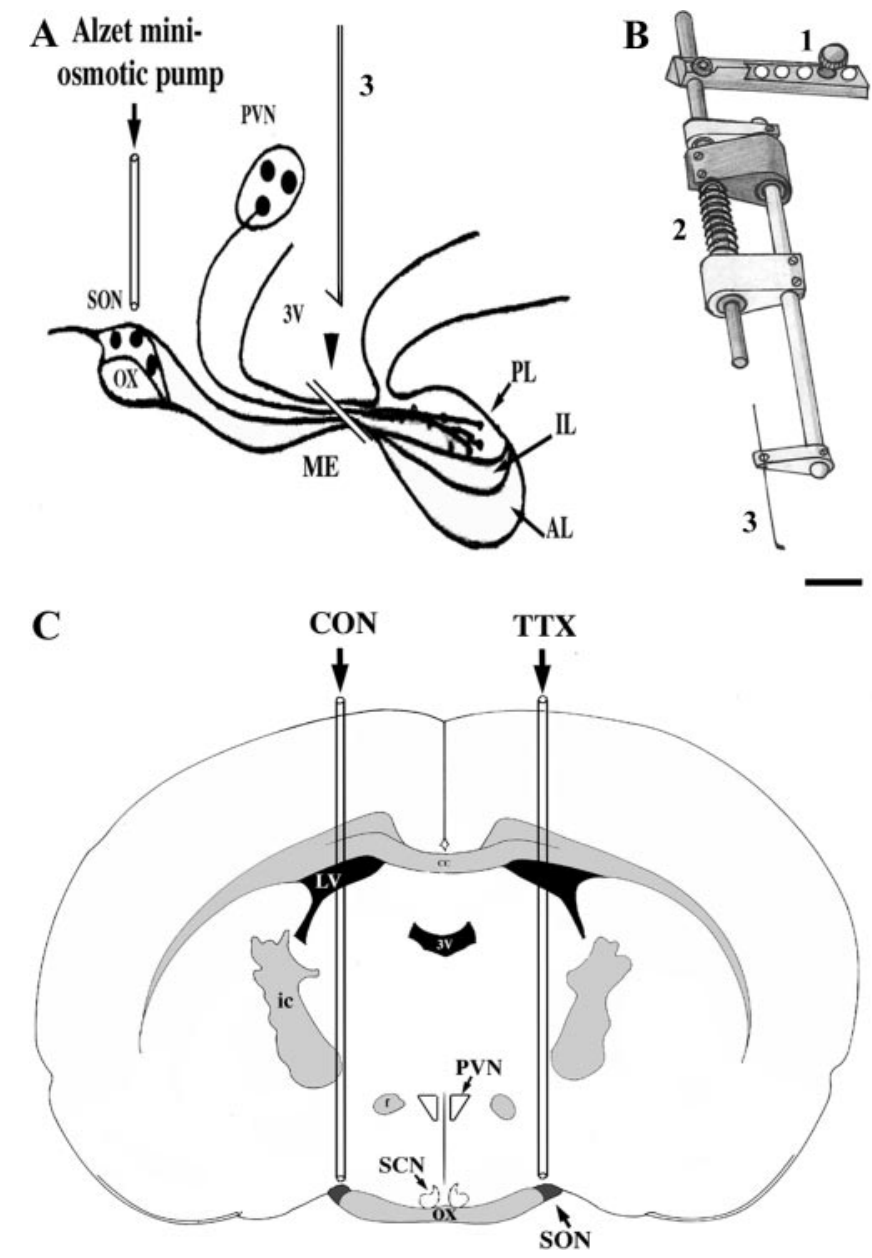

Figure 1. The in vivo experimental system. $A$, Parasagittal view of the HNS in rat brain illustrating the location of the axonal compression injury at the level of the ME (arrowhead) using L-shaped wire (labeled 3) and the placement of the infusion cannulas over the SON. B, Compression device for stereotaxic instrument. The figure shows a three-dimensional view of a specially designed tool for compression of brain structures located over the base of the skull. The compression device was connected to a stereotaxic apparatus (through the bar labeled 1), and the L-shaped wire (labeled 3) was lowered to the level of the ME; by further lowering the wire, a controlled compression could be maintained on the ME. The magnitude of the compression was determined by the strength of the spring (labeled 2). All of the fixed points on the device are illustrated by the positions of the screws. Scale bar, $10 \mathrm{~mm}$. ( shows a rat brain coronal section at the level of mid-SON, demonstrating the location of the bilateral cannulas positioned $\sim 300$ $\mu \mathrm{m}$ over the SON. Cannulas were connected to Alzet miniature osmotic pumps (model 2002) via PE100 tubes, and $3 \mu \mathrm{m}$ TTX was delivered to the experimental side and only vehicle to the control side for 2 weeks. 3V, Third ventricle; $\mathrm{AL}$, anterior lobe; $\mathrm{CON}$, control; ic, internal capsule; $\mathrm{IL}$, intermediate lobe; LV, lateral ventricle; ox, optic chiasm; PL, posterior lobe.

The axonal compression used here was achieved by applying controllable and continuous pressure on the ME using a specially designed tool (Fig. $1 B$ ) (see Materials and Methods) connected to the stereotaxic apparatus. Using a blunted L-shape wire (Fig. $1 A, B)$ connected to the compression device, we could avoid vasculature damage and still produce selective damage to the axons in the ME in a simple and reproducible manner.

\section{Effectiveness of ME compression to produce MCN cell death}

To evaluate the efficacy of the ME compression procedure used here to produce MCN degeneration in the absence of intracerebral cannulation $14 \mathrm{~d}$ after the lesion, the rats were anesthetized with pentobarbital sodium (50 mg/kg, i.p., body weight) and perfused transcardially, and the brains were removed and pro- 
Table 1. Effect of axonal compression on magnocellular neuronal survival in the SON

\begin{tabular}{lll}
\hline Experimental conditions & $\begin{array}{l}\text { Number of VP MCNs per } \\
\text { unilateral SON }\end{array}$ & $\begin{array}{l}\text { Number of OT MCNs per } \\
\text { unilateral SON }\end{array}$ \\
\hline Controls $(n=10)$ & $120 \pm 5$ & $102 \pm 4$ \\
Axonal compression $(n=6)$ & $20 \pm 2^{a}$ & $69 \pm 4^{a}$ \\
\hline
\end{tabular}

Axons were injured by compression for 5 min at the level of the median eminence (see Materials and Methods). Data are expressed as mean \pm SEM of numbers of MCNs counted in unilateral SONs. In all cases, the values shown represent the total numbers of MCNs in five defined coronal sections from a single SON (see Materials and Methods). $n$ is equal to the number of unilateral SONs used for each group.

${ }^{a} p$ values of $<0.05$ for SONs undergoing axonal compression compared with sham controls, which were calculated by one-way ANOVA, followed by a Fisher's PLSD post hoc test.

cessed as described in Materials and Methods. The results showed that the compression alone produced an $83 \%$ decrease in VP MCNs and a $32 \%$ decrease in OT MCNs in the SON compared with the unlesioned control rat SONs (Table 1). The extent of this MCN cell death found in the SON after axonal compression in the ME is comparable with the $65 \%$ decrease in VP MCNs and a $31 \%$ decrease in OT MCNs in the SON found by Dohanics et al. (1996) after compressing the neurohypohysial stalk. The slightly greater cell death found in the VP MCNs in our experiments may be attributable to the more proximal site of the injury. The greater vulnerability of the VP MCNs versus the OT MCNs to the axotomy-induced cell death in our experiments (Table 1) is in agreement with other reports in which different modes of axonal injury were used (Herman et al., 1987; Dohanics et al., 1992, 1996; Alonso et al., 1996). In addition, we observed the expected polydipsia and polyuria in the ME lesioned rats (data not shown), typical of the diabetes insipidus found after injuries to the neurohypophysial axons (O'Connor, 1952; Moll, 1962; Dellmann, 1973; Raisman, 1973; Daniel and Prichard, 1975; Dellmann et al., 1988).

To inhibit the neuronal activity of the MCNs in the SONs of the lesioned animals, we implanted bilateral intracerebral cannulas above the SONs of the lesioned animals (Fig. 1A,C), which were then connected to Alzet miniature osmotic pumps. For a period of 2 weeks, $3 \mu \mathrm{M}$ TTX was delivered to the area of the right SON and vehicle only to the left SON. In this way, each experimental animal provided both a TTX-treated (with inhibited neural activity) and a control SON for analysis.

\section{TTX infusion blocks synaptic activity in the treated unilateral SON but not in the contralateral SON}

Before using the above TTX paradigm in ME-lesioned animals, we first evaluated whether the superfusion of $3 \mu \mathrm{M}$ TTX through the cannula for the $14 \mathrm{~d}$ was adequate to inhibit neural activity in the SON in vivo. It is well known that intraperitoneal injection of hypertonic saline $(0.6 \mathrm{M} \mathrm{NaCl}, 1 \mathrm{ml} / 100 \mathrm{gm})$ elicits strong c-fos activation in MCNs in the SON 90 min after the injection (Ceccatelli et al., 1989; Giovannelli et al., 1990; Sharp et al., 1991; Shiromani et al., 1995). In our preliminary experiments on normal rats, Fos, a marker of neural activity in the SON, was found to be expressed bilaterally and robustly in the SON and in the VP as well as in OT MCNs after this osmotic stimulation. We used this physiologic stimulation to evaluate the efficacy of the unilateral superfusion of $3 \mu \mathrm{M}$ TTX to inhibit neural activity by determining whether the Fos marker of neural activity in the SON was inhibited after $14 \mathrm{~d}$ of infusion of $3 \mu \mathrm{M}$ TTX. Rats $(n=4)$ with bilateral intracerebral cannula implants over the SON but without ME lesions were prepared in which the right SON was infused with $3 \mu \mathrm{M}$ TTX and the left SON with vehicle (PBS) for only 2 weeks. At the end of this period, the rats were osmotically stressed
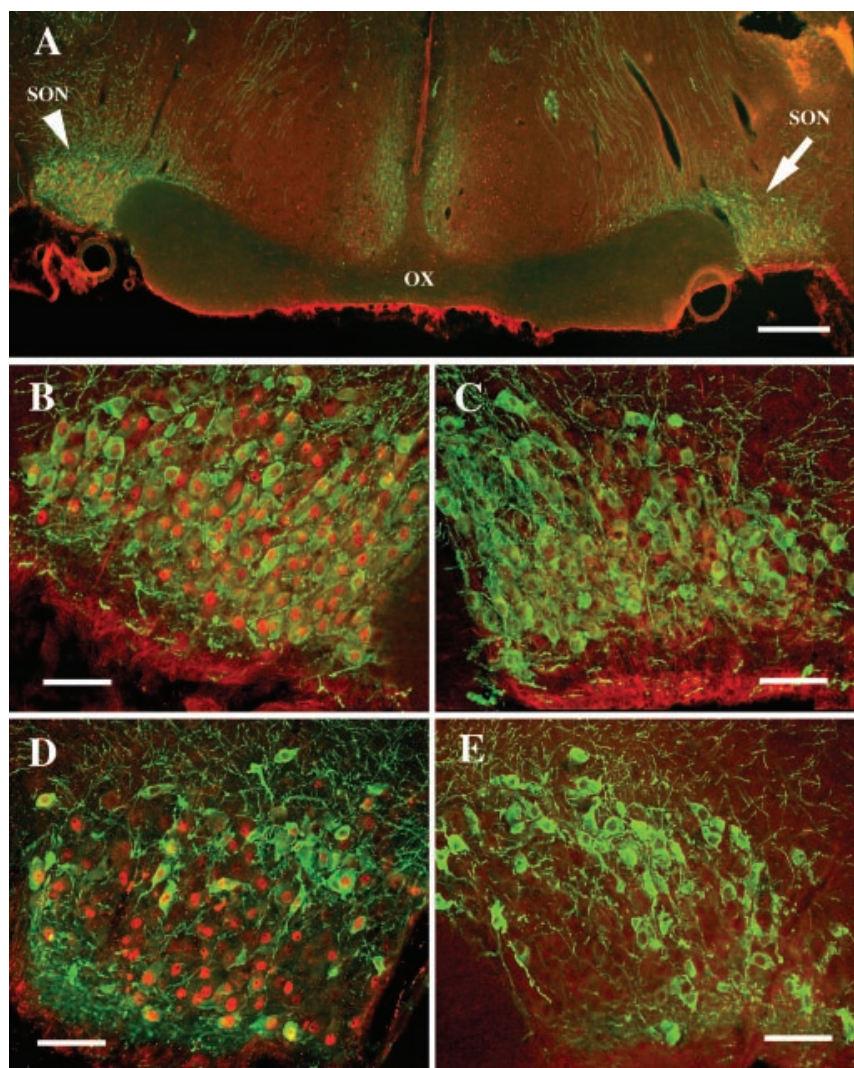

Figure 2. TTX infusion effectively blocks synaptically evoked c-Fos in the MCNs of the SON after osmotic stimulation in vivo. A illustrates a low-magnification coronal view of the SON visualized by double immunofluorescence immunohistochemistry demonstrating VP-NP (green) and c-fos (red) immunoreactivity. One side of the SON (arrow) was infused for $14 \mathrm{~d}$ with $3 \mu \mathrm{m} \mathrm{TTX}$, whereas the control side (arrowhead) was infused with vehicle only. Note that there is an intense punctuate c-fos activation in the untreated SON (arrowhead) but no significant c-Fos activation in the TTX-treated SON (arrow) after osmotic stimulation. $B, C$, Higher magnification of the control $(B$ ) and TTX-treated ( $C$ SONs shown in A.D, E, Double-label immunohistochemistry of OT-NP labeled in green and c-Fos labeled in red in control $(D)$ and in TTX-treated (E) SONs in an adjacent section. OX, Optic chiasm. Scale bars: $A, B, 200 \mu \mathrm{m} ; C, D, 100 \mu \mathrm{m}$.

by an intraperitoneal injection of the $0.6 \mathrm{M} \mathrm{NaCl}$ and then killed 90 min later. The brains were removed and processed for Fos and VP-NP or OT-NP double immunofluorescence.

The results showed strong Fos-positive MNCs in the control, left SON infused with vehicle only (Fig. $2 A$, arrowhead, $B, D$ ), whereas the $3 \mu \mathrm{M}$ TTX-treated side was absolutely Fos negative (Fig. 2A, arrow, $C, E$ ). When the SON sections were doublestained for Fos protein and VP-NP or OT-NP, we found that both the VP neurons (Fig. 2B) and the OT neurons (Fig. 2D) were Fos positive but only in the control (vehicle only) SON. Given these data, we concluded that the infusion of $3 \mu \mathrm{M}$ TTX through the SON completely blocked the neural activation of the SON after osmotic stress, and that the superfusion procedure was effective even $14 \mathrm{~d}$ after implantation of the Alzet miniature osmotic pumps.

\section{Inhibition of neural activity by TTX reduces the survival of} MCNs in the SON after ME compression

Before examining whether there was a direct effect of TTX superfusion on the MCN survival after axonal compression, we first evaluated the effect of the TTX superfusion alone on MCN survival. In these experiments, we studied rats without ME compression but those in which $3 \mu \mathrm{M}$ TTX was infused into the right SON and vehicle only into the left SON via the intracerebral cannulas. 
Table 2. Effect of axonal injury and unilateral TTX infusion on MCN survival in the SON

\begin{tabular}{|c|c|c|c|c|}
\hline \multirow[b]{2}{*}{ Experimental conditions } & \multicolumn{2}{|c|}{ Number of VP MCNs per unilateral SON } & \multicolumn{2}{|c|}{ Number of OT MCNs per unilateral SON } \\
\hline & Vehicle only & Plus TTX & Vehicle only & Plus TTX \\
\hline Control $(n=5)$ & $137 \pm 211$ & $132 \pm 16$ & $88 \pm 8$ & $85 \pm 7$ \\
\hline ME compression $(n=5)$ & $32 \pm 11$ & $18 \pm 6$ & $55 \pm 3$ & $30 \pm 2^{a}$ \\
\hline
\end{tabular}

In these studies, both controls and axonally injured rats contained bilateral cannulas over their SONs, which delivered either PBS (vehicle) or $3 \mu \mathrm{m}$ TTX in PBS (see Materials and Methods). Data are expressed as mean \pm SEM numbers of MCN counted in unilateral SONs. $n$ is equal to the number of unilateral SONs from separate rats.

${ }^{a} p$ values of $<0.05$ between vehicle only-infused versus Plus TTX-treated rats, which were calculated by one-way ANOVA, followed by a Fisher's PLSD post hoc test.
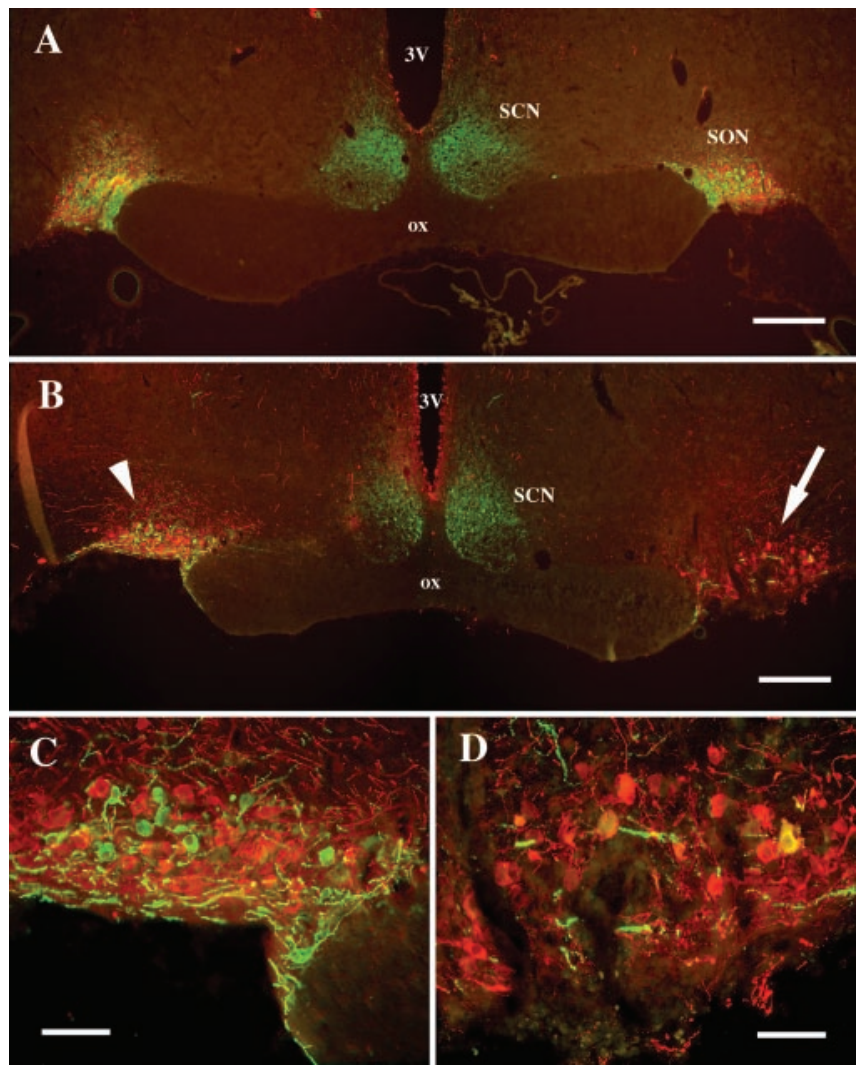

Figure 3. TTX inhibition of neuronal activity in the SON reduces survival of the MCNs after axonal injury. Double-label immunofluorescence for VP-NP (green) and OT-NP (red) of MCNs in the SON was performed $14 \mathrm{~d}$ after ME compression injury and continuous infusion with either control (vehicle) or $3 \mu \mathrm{m}$ TTX over the left and right SONs, respectively, for 2 weeks. A shows a low-magnification view of the control condition, with no axonal injury and bilateral infusion of vehicle via the implanted intracerebral cannulas connected to the mini osmotic pumps for the entire 2 weeks. $B$ shows a similar low-magnification double-immun ofluorescent view of immunoreactive VP-NP (green) and OT-NP (red) MCNs in rat hypothalamic SON 2 weeks after ME transection and with continuous unilateral infusion of TTX (arrow) or vehicle only (arrowhead) over the 2 weeks. ME transection itself leads to neurodegeneration of both VP and OT neurons of the SON (compare left side in $A$ with left side in $B$ ) (Table 1). However, at higher magnification $(C, D)$, it can be seen that the infusion of TTX in the SON area after axonal injury leads to greater cell death of the MCNs in TTX ( $D$ ) as opposed to when only vehicle is infused ( $C$ ). See Table 1 for quantitative analysis. Scale bars: $A, B, 400 \mu \mathrm{m} ; C, D, 100 \mu \mathrm{m}$.

Fourteen days after surgery, rats were killed by transcardial perfusion and the brains were removed, processed, and evaluated by immunohistochemistry. We found no differences in MNC number in either the OT or VP MNCs between the TTX-treated SON and the vehicle only-treated SONs in the absence of ME compression (Table 2, control).

In contrast, when we compared the SONs in the $3 \mu \mathrm{M}$ TTXtreated side (Fig. $3 B$, arrow, $D$ ) with the SONs in the vehicle onlytreated side (Fig. 3B, arrowhead, $C$ ) in the rats that did undergo $\mathrm{ME}$ compression, we found that the MNC neurons were clearly disrupted by the TTX treatment (Fig. 3C,D). Furthermore, in the
ME compression group, both the OT and VP MNCs decreased in number as a result of the TTX treatment (Table 2). The VP MNCs fell from a representative value of 32 in the control SON to 18 in the TTX-treated SON, and the OT MNCs fell from 55 in the control SON to 30 in the TTX-treated SON in the axonal compression group (Table 2). From these data, we can conclude at least for the OT MNCs that the block of neural activity by $3 \mu \mathrm{M}$ TTX significantly increased the neurodegeneration process caused by the ME compression. The decrease in the VP MCNs, although in the same direction as for the OT MCNs, was not statistically significant, in part because of the large decrease already present after the axonal compression even in the absence of TTX.

In view of these findings, we also made an attempt to rescue the SON MCNs after ME compression by unilateral superfusion of $\mathrm{KCl}$ into the rat $\mathrm{SON}$ through an intracerebral cannula. The continuous infusion of $50 \mathrm{~mm} \mathrm{KCl}$ over the SON for a 2 week period after ME compression in either the absence or presence of TTX led to an unacceptable rate of morbidity and mortality among the treated rats. For this reason, we decided to perform this experiment in vitro by using the hypothalamic slice culture experimental model. By using this in vitro approach, we could confirm the above experimental results obtained with TTX in vivo and could also study the effects of potassium depolarization on MCN survival after axonal injury.

\section{The in vitro model}

The organotypic method of slice culture allows for preservation of the rat hypothalamus and the topographic organization of its nuclei (House et al., 1998). Figure $4 A-C$ shows the localization of the SON, paraventricular hypothalamic nucleus (PVN), accessory nuclei (ACC), and SCN after immunostaining for total NP after $15 \mathrm{~d}$ of culture. Figure 4 also shows higher-power views of the SONs stained for total NP-immunoreactive cells (Fig. 4D) and more specifically for OT-associated NP-immunoreactive cells (Fig. 4E) and VP-associated NP-immunoreactive cells (Fig. $4 F$ ). These immunoreactive neurons are similar in their morphological appearances to MCNs found in the SON in vivo (Armstrong, 1995). Although the MCN phenotypes are present in these cultures, it has also been shown that there is substantial programmed cell death of the MCN cell population in these slices (Vutskits et al., 1998; Rusnak et al., 2002, 2003). This is presumably because of the extensive axotomy that these neurons undergo during preparation of the slices. Previous studies have focused on the ability of the neurotrophic factor CNTF to counter this cell death in vitro (Vutskits et al., 1998; Rusnak et al., 2002, 2003).

\section{The effects of TTX and $\mathrm{KCl}$ on MCN survival after axotomy}

We used the organotypic culture model system to further examine the influence of neural activity in the survival of MCNs in the SON after this form of axotomy-induced neural degeneration. TTX was used to block the spontaneous neuronal activity in the culture (Wray et al., 1991), and $\mathrm{KCl}$ depolarization was used to 


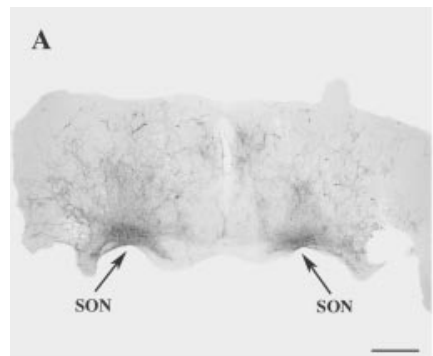

B
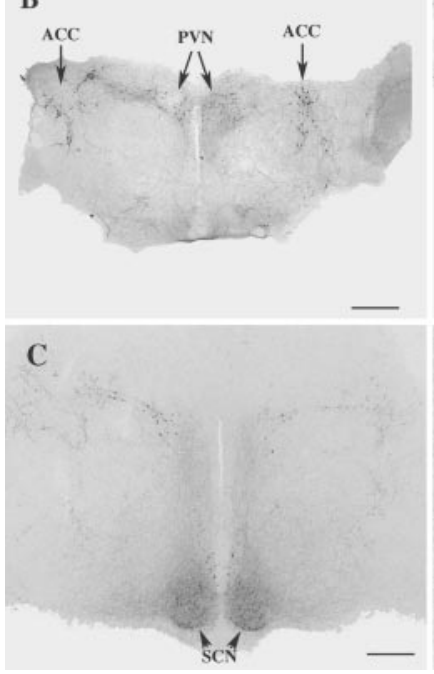
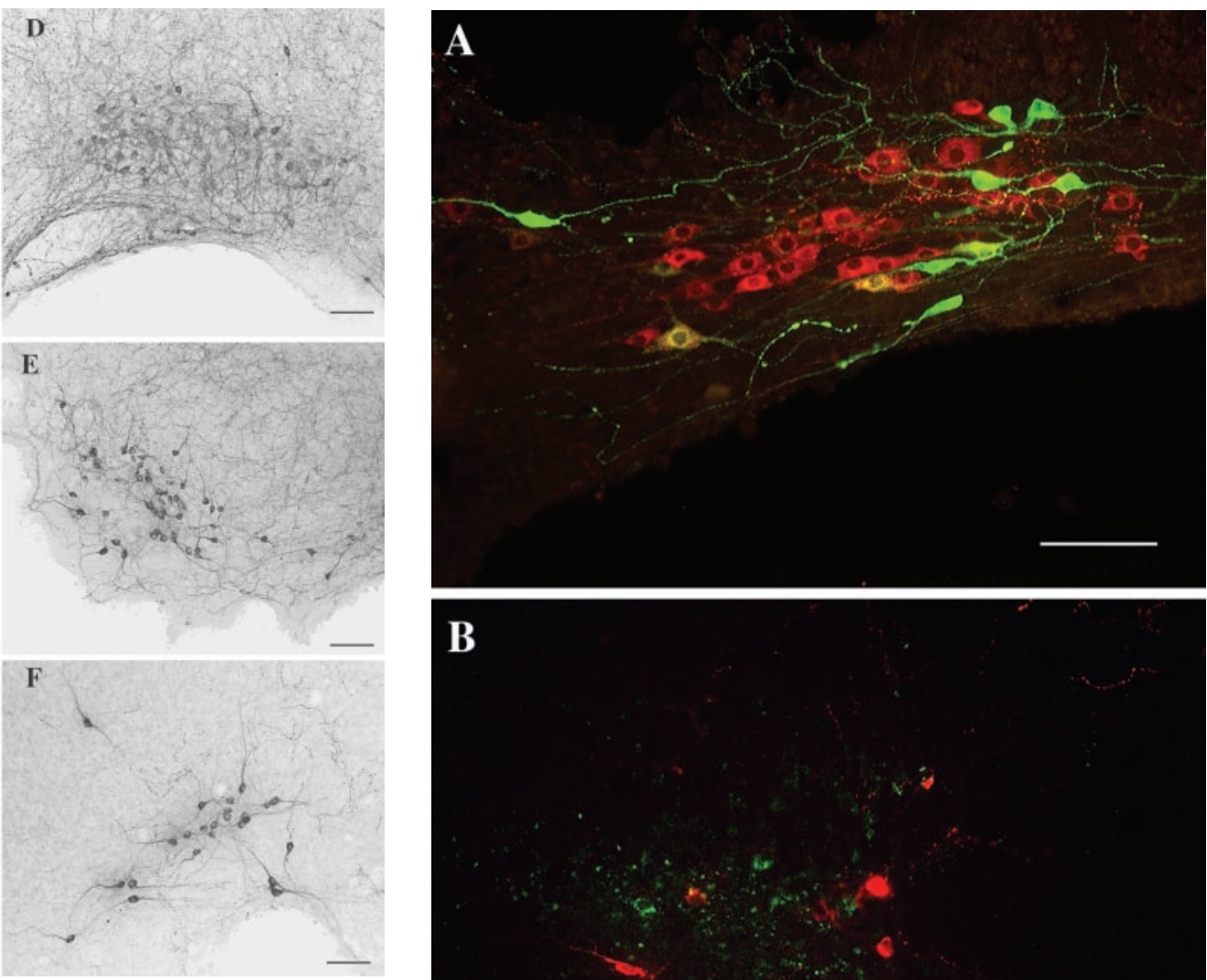

Figure 4. The in vitro experimental system: representative views of VP and OT magnocellular neurons in the SON in rat hypothalamic slices, which were cultured for $15 \mathrm{~d}$ and visualized by the avidin-biotin immunohistochemical technique. $A-C$, Note that in the low-magnification view, there is preservation of the SON, PVN, and SCN when these nuclei are visualized using a panspecific anti-NP, PS45, as the primary antibody. $D-F$, Higher-magnification views $(D, E)$ show the well differentiated MCN morphology seen in the SON visualized by anti-NP-PS45 (D), antiOT-NP-PS38 ( $E$ ), and anti-VP-NP-PS41 ( $F$ ) antibodies. Scale bars: $A-C, 800 \mu \mathrm{m} ; D-F, 100 \mu \mathrm{m}$.

mimick enhanced neural activity. We found that the addition of $2.5 \mu \mathrm{M}$ TTX to the culture media produced a large decrease in the numbers of OT and VP MCNs surviving after $15 \mathrm{~d}$ of culture (Fig. $5 A, B)$. The number of VP MCNs in TTX fell by $70 \%$ compared with the controls (Fig. 6A), and the number of OT MCNs in TTX decreased by $76 \%$ compared with the controls (Fig. $6 B$ ). In contrast, when $25 \mathrm{~mm} \mathrm{KCl}$ was present in the culture medium for $15 \mathrm{~d}$, this increased the survival of the MCNs in the rat SON slice cultures (Fig. $5 A, C$ ) by $97 \%$ for the VP MCNs (Fig. 6A) and by $134 \%$ for OT MCNs (Fig. $6 B$ ).

\section{The rescue of the MCNs from TTX enhanced neurodegeneration after axotomy}

Given the deleterious effect of TTX on the survival of the MCNs in vitro, we examined the possibility of reversing its effects by $\mathrm{KCl}$ depolarization of the MCNs in the TTX-treated cultures. Figure 7 shows that TTX caused a profound loss of both OT and VP MCNs (Fig. 7, compare $A$ and $B$ with $C$ and $D$ ) and that there was a significant rescue of both MCN subtypes from TTX-induced cell death by $\mathrm{KCl}$ depolarization (Fig. $7 E, F$ ). A quantitative analysis of these experiments is presented in Figure 8, which shows that TTX plus $\mathrm{KCl}$ increases the number of VP MCNs by $97 \%$ and the number of the OT MCNs by $354 \%$ when compared with the TTX only-treated slice culture. The addition of CNTF to the TTXtreated slice culture for a period of $15 \mathrm{~d}$ led to an even larger reversal of the TTX effect in both the VP and OT MCNs, where
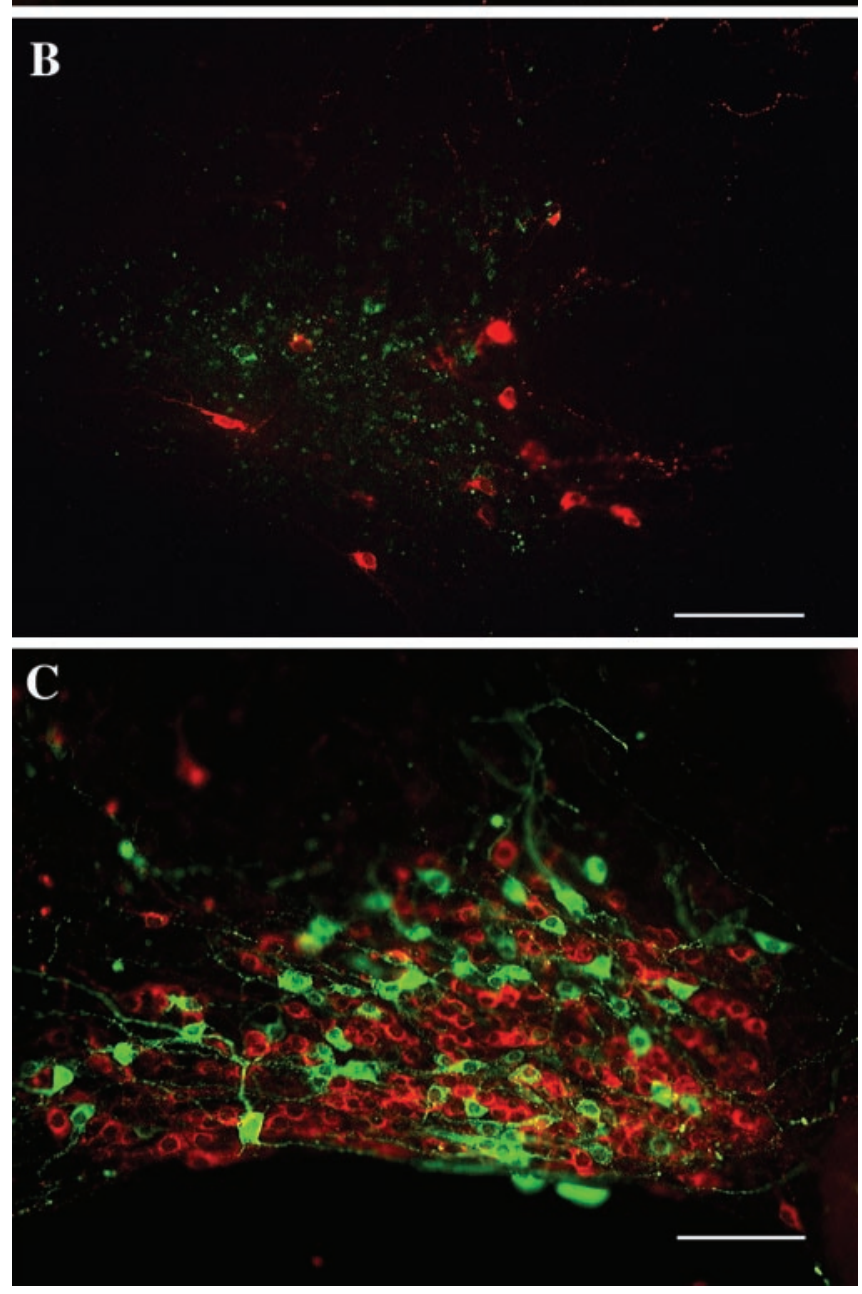

Figure 5. Survival of MCNs in the rat hypothalamic slice culture is affected by neuronal activity. A-C, Representative double-immunofluorescent views of immunoreactive 0T-NP (red) and VP-NP (green) MCNs in rat hypothalamic SON slices cultured for $15 \mathrm{~d}$ in control media $(A)$, in the presence of $2.5 \mu \mathrm{M} T \mathrm{TTX}(B)$, or in the presence of $25 \mathrm{~mm} \mathrm{KCl}(C)$. The addition of TTX to the culture media led to extensive cell death of VP and $0 \mathrm{~T}(B)$, whereas the depolarizing agent $\mathrm{KCl}$ significantly improved the survival of both the VP and OT MCNs ( $C$ ) in vitro. Scale bar, $100 \mu \mathrm{m}$.

the TTX plus CNTF increases the number of VP MCNs by $426 \%$ and the OT MCN number increased by $669 \%$ when compared with the TTX-only group. When the $\mathrm{KCl}$ and $\mathrm{CNTF}$ were added together to the TTX-treated slice cultures, we found that the VP MCN number increased by $558 \%$ and the OT MCN number was 

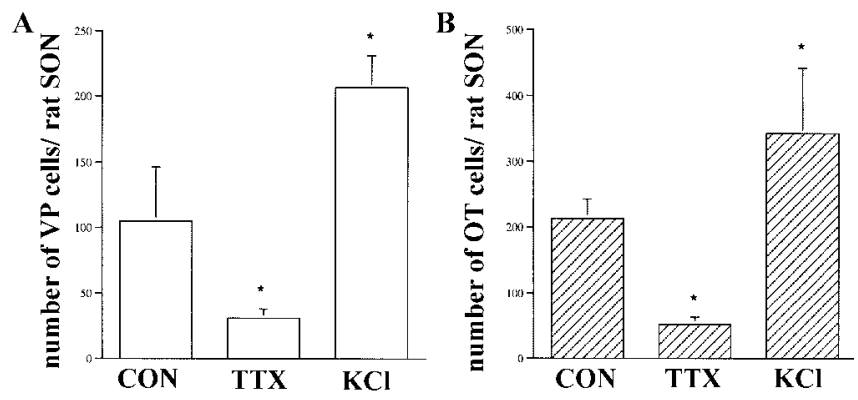

Figure 6. Quantification of the effect of culturing hypothalamic slices in the absence of any treatment (CON), in the presence of $2.5 \mu \mathrm{MTTX}$, or in the presence of $2.5 \mu \mathrm{m} \mathrm{KCl}$ on the number of surviving VP-NP $(A)$ and OT-NP $(B)$ immunoreactive MCNs present in cultures of rat SONs. Data are expressed as mean \pm SEM, where $n=12-22$ per group. Asterisks represent $p$ values of $<0.05$ (compared with control) and were calculated by one-way ANOVA followed by Fisher's PLSD post hoc test.
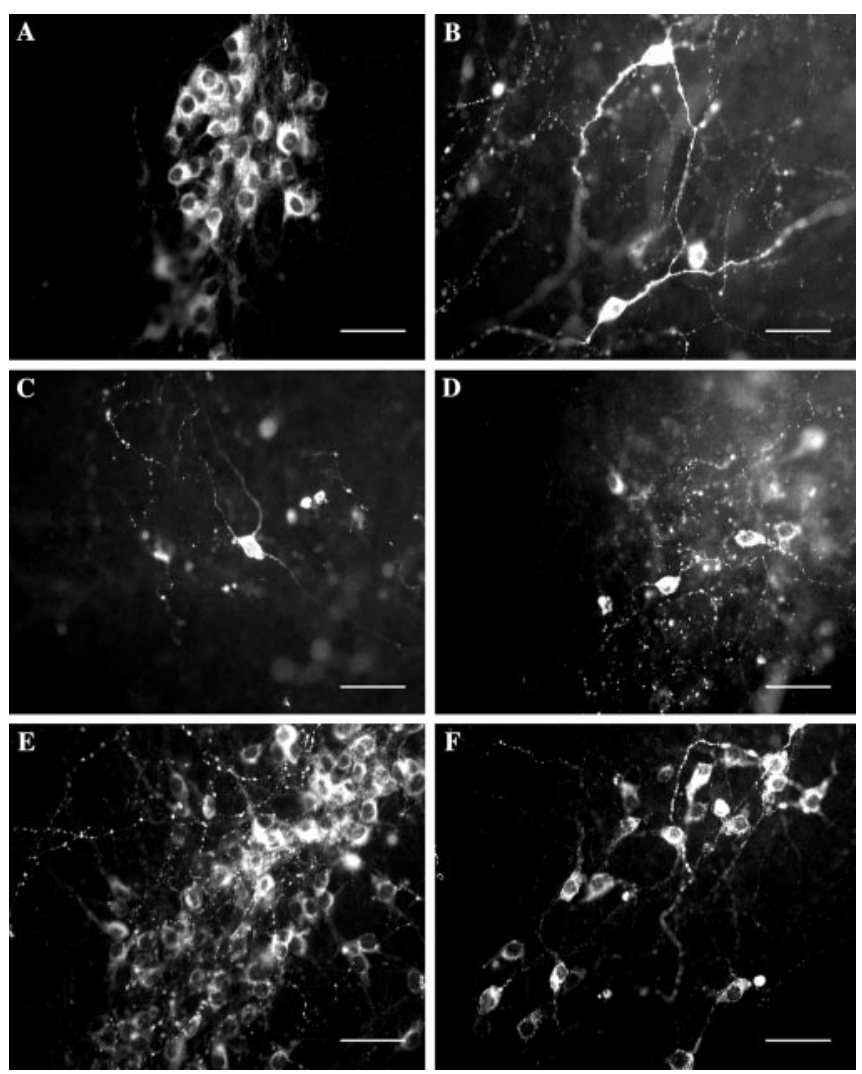

Figure 7. The deleterious effect of TTX on MCN survival in vitro is reversed by coincubation in $25 \mathrm{~mm} \mathrm{KCl}$. $A-F$, Representative immunofluorescent views of immunoreactive 0T-NP $(A, C, E)$ and VP-NP $(B, D, F)$ MCNs in the SON in rat hypothalamic slices cultured for $15 \mathrm{~d}$. Control cultured SONs in the absence of any treatment $(A, B)$, SONs cultured in the presence of $2.5 \mu \mathrm{m}$ TTX (C, D), and SONs cultured in the presence of both $2.5 \mu \mathrm{M}$ TTX and $25 \mathrm{~mm} \mathrm{KCl}(E, F)$ are shown. Note the low number of surviving cells and degenerating neurites in the TTX $(C, D)$ and the reversal of this effect when the depolarizing agent $\mathrm{KCl}$ was coincubated with TTX. Scale bar, $50 \mu \mathrm{m}$.

found to increase by $754 \%$ compared with the TTX-only group. These data indicate that although the potassium depolarization and the CNTF were effective in reversing the TTX-induced MCN death, the CNTF treatment was more efficacious, especially for the VP MCNs (Fig. 8).

\section{Effects of $\mathrm{KCl}$ and TTX on neurite density}

Figure 9 illustrates OT and VP MCN neurites in slice cultures that were incubated in $25 \mathrm{mM} \mathrm{KCl}, 2.5 \mu \mathrm{M}$ TTX, or a combination of

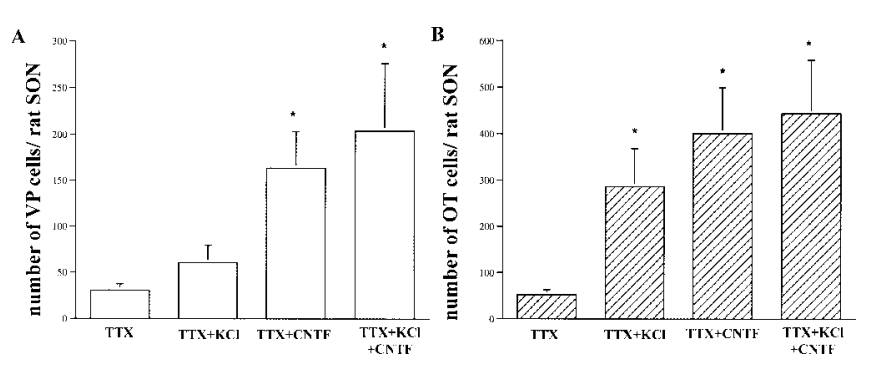

Figure 8. $A, B$, Quantification of the effect of culturing hypothalamic slices in the presence of $2.5 \mu \mathrm{m}$ TTX alone or with the addition of $25 \mathrm{~mm} \mathrm{KCl}$ or the neurotrophic factor CNTF (10 mg/ml) on the number of surviving VP $(A)$ and $0 \mathrm{~T}(B)$ MCNs present in cultures of rat SONs. The conditions used were TTX alone, TTX plus KCl, TTX plus CNTF, and TTX plus KCL plus CNTF. Data are expressed as mean \pm SEM, where $n=7-22$ per group. Asterisks represent $p$ values of $<0.05$ (compared with control) and were calculated by one-way ANOVA followed by Fisher's PLSD post hoc test.

both. There was a much higher neurite density in the KCl-treated slice cultures (Fig. 9C,D) and a much lower density of neurites in the TTX-treated slice cultures (Fig. 9E,F) compared with the control group (Fig. 9A,B). When TTX is present with $25 \mathrm{~mm} \mathrm{KCl}$, the $\mathrm{KCl}$ treatment clearly reversed the deleterious effect of the TTX (Fig. 9G,H).

\section{Discussion}

In this study, we directly examined whether the inhibition of neural activity in the SON by TTX can affect the survival of MCNs after axonal injury in the HNS in vivo and in vitro. Previous studies in vivo using experimental treatments that are inferred to reduce excitatory input to the HNS (Herman et al., 1986, 1987; Dohanics et al., 1996) found that this produced a substantial decrease in MCN survival after HNS axon injury. The reverse treatment, chronic hypernatremia, did not alter the survival of the axotomized MCNs in one study (Dohanics et al., 1996); however, in another study that used a chronic intermittent saltloading paradigm, there was an increase in the survival of MCN cells (Huang and Dellmann, 1996). In view of these reports, we tested the hypothesis that after axotomy, neural activity was neuroprotective in the HNS by directly inhibiting both electrical and synaptic activity unilaterally in the SON by local superfusion of TTX (Ludwig et al., 1995) in vivo. Each animal's contralateral SON served as the (noninhibited) control in these experiments.

The results of this experiment in vivo supported the hypothesis that there is a neuroprotective role for functional activity in the HNS. The TTX superfusion that we used for 2 weeks was effective in blocking evoked excitatory input to the SON (Fig. 2) but did not by itself alter MCN number or the structure of the SON in the absence of axonal compression (Table 2, control). However, it did cause considerable disruption of cellular organization (Fig. $3 C, D)$ and a decrease in MCN number in the axotomized and TTX-treated SON (Table 2, axonal compression). These data are comparable with those reported by Dohanics et al. (1996), who found that axonal compression alone caused a decrease in VP and OT MCNs to $\sim 65$ and $31 \%$ of unlesioned control SON values, but that together with chronic hyponatremia, it produced a 97 and $90 \%$ decrease, respectively. Similarly, axonal compression alone in our studies produced a 76\% decrease in VP MCNs and a $37 \%$ decrease in OT MCNs (Table 2), which then fell to an $87 \%$ decrease in VP MCNs and a 65\% decrease in OT MCNs with the TTX treatment, compared with unlesioned control SONs (Table 2 ). Thus, these data obtained using the TTX paradigm provide direct evidence in vivo for the role of neural activity in the survival of MCNs after axonal injury by ME compression and show that 

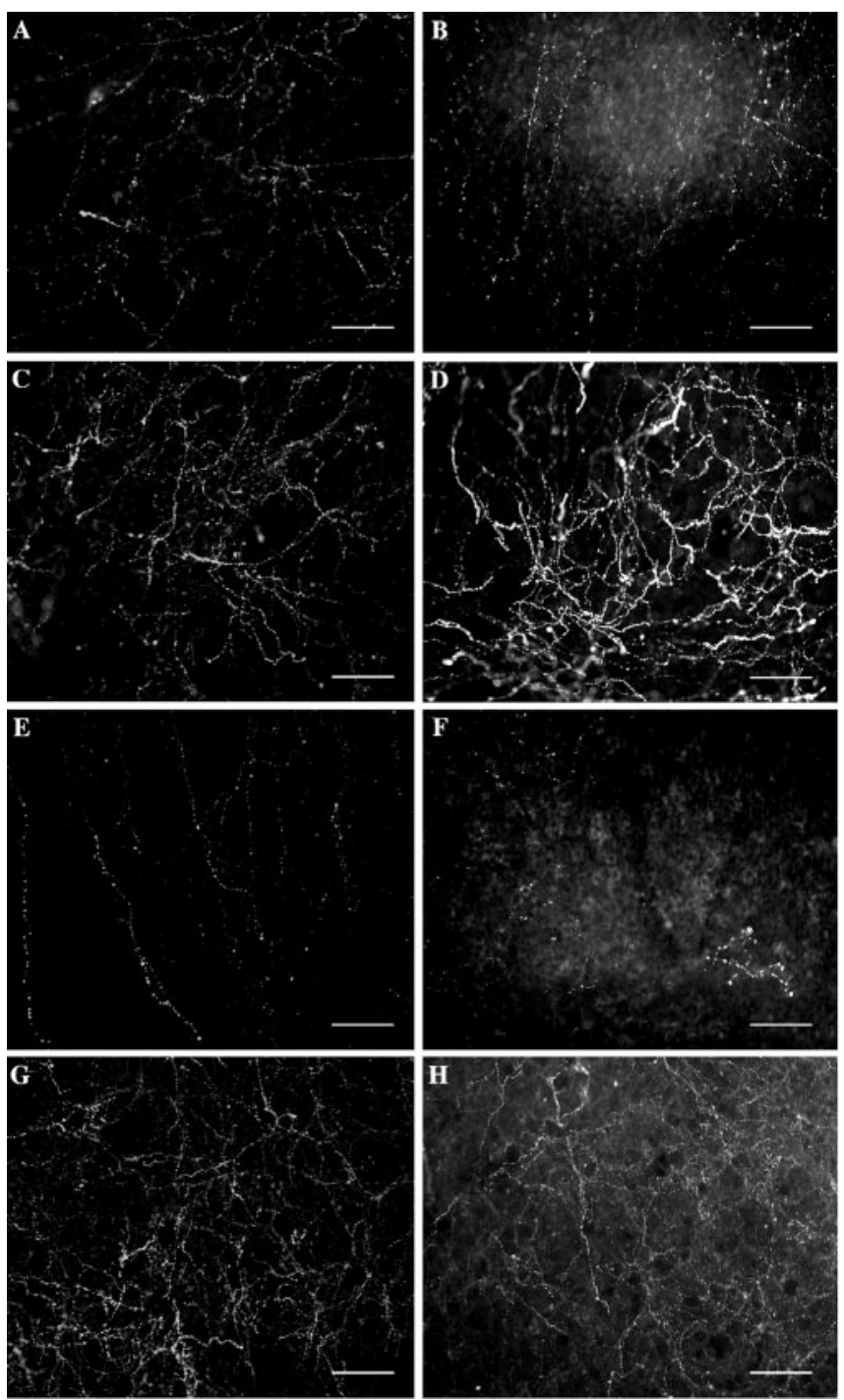

Figure 9. Effects of $\mathrm{KCl}$ and TTX on MCN neurite density in vitro. $A-H$, Representative immunohistochemical views of immunoreactive $0 T(A, C, E, G)$ and $V P(B, D, F, H)$ MCN fibers found adjacent to the $S O N$ in rat hypothalamic slices cultured for $15 \mathrm{~d}$. Control $S O \mathrm{~N}$ slices cultured in the absence of any treatment $(A, B)$, SONs cultured in the presence of $25 \mathrm{mmKCI}(C, D)$, SONs cultured in the presence of $2.5 \mu \mathrm{m} T \mathrm{TX}(E, F)$, and SONs cultured in the presence of both $2.5 \mu \mathrm{m} T \mathrm{TX}$ and $25 \mathrm{~mm} \mathrm{KCl}$ $(G, H)$ are shown. Scale bar, $100 \mu \mathrm{m}$. Note that the deleterious effect of TTX on MCN neurite outgrowth $(C, D)$ was reversed by the simultaneous application of $K C l$ in the media $(G, H)$.

block of this activity accentuates the process of retrograde degeneration.

In addition to confirming the in vivo data using TTX inhibition, the in vitro experiments in this study also extended the experiments by attempts to rescue the MCNs from axonal injury. Organotypic slice-explant cultures of rat hypothalamus are in vitro models for the study of axotomized MCNs. In the process of preparing these cultures, coronal cuts are made through the hypothalamus that transect all of the MCN axons projecting to the neurohypophysis. This is essentially equivalent to ME transection and neurohypophysectomy in vivo and produces substantial retrograde degeneration of the MCNs both in the SON and in the PVN in vitro (Vutskits et al., 1998; Rusnak et al., 2002, 2003). That this degeneration of the MCNs in vitro is attributable primarily to the axotomy and not to a general excitotoxicity caused by the damage to slice preparation is suggested by findings that the MCNs are relatively resistant to glutamate excitotoxicity and ischemia (Curras-Collazo et al., 2002). When $2.5 \mu \mathrm{M}$ TTX was added to the culture medium, there was a large increase in cell death of the VP and OT MCNs in the cultured SONs (Figs. 5, 6), indicating that, as in vivo, the survival of axotomized MCNs was detrimentally affected by the inhibition by TTX of the spontaneous electrical and synaptic activity that is typically found in these cultures (Wray et al., 1991).

The cell death of the MCNs in vitro has been attributed to apoptosis (Vutskits et al., 1998), and it is well known that increasing extracellular $\mathrm{K}^{+}$can block apoptosis in a great variety of neurons and promotes their survival in the absence of neurotrophic factors in the culture media. Elevation of extracellular $\mathrm{KCl}$ has been demonstrated to promote the survival of a wide variety of peripheral and central neurons (Gallo et al., 1987; Collins and Lile, 1989; Koike et al., 1989; Collins et al., 1991; Johnson et al., 1992; Galli et al., 1995; Pike et al., 1996; Yu et al., 1997; Colom et al., 1998). When the hypothalamic organotypic cultures were incubated in $25 \mathrm{~mm} \mathrm{KCl}$, there was a considerable improvement in survival of the OT and VP MCNs (Figs. 5, 6), indicating that the potassium depolarization was able to counter the effects of axotomy. In addition, we observed that the deleterious effects of TTX on MCN survival in vitro could be reversed by potassium depolarization (particularly for the OT MCNs) and even more dramatically for both MCN subtypes by the neurotrophic factor CNTF (Fig. 8).

The above experiments indicate that both neural activity and neurotrophic factors are neuroprotective for the MCNs in response to axonal injury. Consistent with the "neurotrophic hypothesis," which posits that the survival of neurons depends on the continuous retrograde supply of trophic factors from target organs (Purves, 1988; Oppenheim, 1989, 1991), Vutskits et al. (1998) proposed that the programmed cell death of MCNs in the HNS is attributable to the loss of specific trophic actors, presumably CNTF, from the neurohypophysis. In this regard, the rescue of the MCNs in vitro by CNTF (Vutskits et al., 1998; Rusnak et al., 2002, 2003) and the reversal of the TTX effect (Fig. 8) provide support for this proposal. The finding in this study that neural activity in vivo and in vitro and potassium depolarization in vitro are neuroprotective in the HNS is also in keeping with similar findings in other neuronal systems in the CNS (Goldberg and Barres, 2000). Neural activity and potassium depolarization have been found to increase the survival of many CNS neuronal types in vitro, including cerebellar granule cells (Gallo et al., 1987; Balazs et al., 1988; Damgaard et al., 1996; DeMello et al., 1997), hippocampal neurons (Barger, 1999), striatal neurons (Nakao, 1998), cortical neurons (Ghosh et al., 1994), retinal ganglion cells (Meyer-Franke et al., 1995, 1998; Goldberg and Barres, 2000), and spinal motor neurons (Hanson et al., 1998; Soler et al., 1998).

The mechanism by which neural activity can alleviate programmed cell death in CNS neurons is not well understood. It is interesting that the axotomy of spinal and hypoglossal motor neurons can cause "synaptic stripping," a mechanism involving microglia, astrocytes, and the postsynaptic neuron, that removes or "strips" away presynaptic buttons (Blinzinger and Kreutzberg, 1968; Svensson and Aldskogius, 1993; Aldskogius et al., 1999). The consequence of this would be to reduce synaptic input to the neuron and thereby reduce the neuroprotective effect of activity in situ. This can be mimicked by the application of TTX and overcome in vitro by the application of increased extracellular $\mathrm{KCl}$ concentrations to depolarize the neurons and, thus, to provide neuroprotection to motorneurons (Soler et al., 1998). However, the mechanism of the survival effect produced by potassium depolarization is controversial. Experiments by Johnson and coworkers 
(Franklin and Johnson, 1992; Johnson et al., 1992) demonstrated that the anti-apoptotic effect of potassium depolarization was caused by an increase in $\mathrm{Ca}^{2+}$ influx through voltage-gated $\mathrm{Ca}^{2+}$ channels, which raised the internal $\mathrm{Ca}^{2+}$ concentration to optimal levels necessary for survival. This view was termed the "calcium set-point hypothesis" (Johnson et al., 1992). Although these data strongly suggested that the increased $\mathrm{Ca}^{2+}$ was responsible for enhanced survival, other studies indicated that there was no correlation between the measured intracellular $\mathrm{Ca}^{2+}$ levels and different membrane potentials induced by different extracellular $\mathrm{K}^{+}$concentrations (Gallo et al., 1987; Collins and Lile, 1989; Koike et al., 1989; Galli et al., 1995; Tong et al., 1997). Indeed, work on various neurons showed that both elevated extracellular $\mathrm{K}^{+}$and $\mathrm{K}^{+}$channel blockers, such as tetraethyl ammonium, suppress apoptosis and appear to do so by preventing decreases in intracellular $\mathrm{K}^{+}$(Yu et al., 1997, 1998, 1999; Colom et al., 1998; Dallaporta et al., 1998; Hughes and Cidlowski, 1999). This protective effect can be independent of changes in $\mathrm{Ca}^{2+}$ (Yu et al., 1997, 1998, 1999).

It is interesting that in a number of neuronal systems, an increase in intracellular cAMP is sufficient to promote and maintain neuronal survival (Hanson et al., 1998; Nakao, 1998). In retinal ganglion cells, it has been shown that both depolarization and increased intracellular cAMP act by recruiting a neurotrophin receptor to the plasma membrane by translocation from intracellular stores (Meyer-Franke et al., 1998; Shen et al., 1999; Goldberg and Barres, 2000), thereby enhancing survival by activating a neurotrophic mechanism. Which, if any, of these mechanisms are evoked by neural activity in the HNS remains to be determined.

\section{References}

Aldskogius H, Liu L, Svensson M (1999) Glial responses to synaptic damage and plasticity. J Neurosci Res 58:33-41.

Alonso G, Bribes E, Chauvet N (1996) Survival and regeneration of neurons of the supraoptic nucleus following surgical transection of neurohypophysial axons depend on the existence of collateral projections of these neurons to the dorsolateral hypothalamus. Brain Res 711:34-43.

Armstrong WE (1995) Morphological and electrophysiological classification of hypothalamic supraoptic nuclei. Prog Neurobiol 47:291-339.

Balazs R, Jorgensen OS, Hack N (1988) N-methyl-D-aspartate promotes the survival of cerebellar granule cells in culture. Neuroscience 27:437-451.

Barger SW (1999) Complex influence of the L-type calcium-channel agonist BayK8644(+/-) on $N$-methyl-D-aspartate responses and neuronal survival. Neuroscience 89:101-108.

Ben-Barak Y, Russell JT, Whitnall MH, Ozato K, Gainer H (1985) Neurophysin in the hypothalamo-neurohypophysial system. I. Production and characterization of monoclonal antibodies. J Neurosci 5:81-97.

Blinzinger K, Kreutzberg G (1968) Displacement of synaptic terminals from regenerating motoneurons by microglial cells. Z Zellforsch Mikrosk Anat $85: 145-157$.

Bodian D, Maran TH (1951) The effect of neuro- and adenohypophysectomy on retrograde degeneration in hypothalamic nuclei of the rat. J Comp Neurol 94:485-514.

Ceccatelli S, Villar MJ, Goldstein M, Hokfelt T (1989) Expression of c-Fos immunoreactivity in transmitter-characterized neurons after stress. Proc Natl Acad Sci USA 86:9569-9573.

Collins F, Lile JD (1989) The role of dihydropyridine-sensitive voltagegated calcium channels in potassium-mediated neuronal survival. Brain Res 502:99-108.

Collins F, Schmidt MF, Guthrie PB, Kater SB (1991) Sustained increase in intracellular calcium promotes neuronal survival. J Neurosci 11:2582-2587.

Colom LV, Diaz ME, Beers DR, Neely A, Xie WJ, Appel SH (1998) Role of potassium channels in amyloid-induced cell death. J Neurochem 70:1925-1934.

Curras-Collazo MC, Patel UB, Hussein MO (2002) Reduced susceptibility of magnocellular neuroendocrine nuclei of the rat hypothalamus to tran- sient focal ischemia produced by middle cerebral artery occlusion. Exp Neurol 178:268-279.

Daniel PM, Prichard MM (1975) Studies of the hypothalamus and pituitary gland with special reference to the effects of tranaction of the pituitary stalk. Acta Endocrinol Suppl (Copenh) 201:1-216.

Dallaporta B, Hirsch T, Susin SA, Zamzami N, Larochette N, Brenner C, Marzo I, Kroemer G (1998) Potassium leakage during the apoptotic degradation phase. J Immunol 160:5605-5615.

Damgaard I, Trenkner E, Sturman JA, Schousboe A (1996) Effect of K+and kainate-mediated depolarization on survival and functional maturation of GABAergic and glutamatergic neurons in cultures of dissociated mouse cerebellum. Neurochem Res 21:267-275.

Daniel PM, Prichard MM (1975) Studies of the hypothalamus and the pituitary gland with special reference to the effects of transection of the pituitary stalk. Acta Endocrinol Suppl (Copenh) 201:1-216.

Dellmann HD (1973) Degeneration and regeneration of neurosecretory systems. Int Rev Cytol 36:215-315.

Dellmann HD, Lue LF, Bellin SI, Ouassat M (1988) An immunohistochemical and fine-structural analysis of peptidergic hypothalamic neurosecretory axon regeneration into the leptomeninges of the rat. Brain Res 450:181-189.

DeMello SR, Borodezt K, Soltoff SP (1997) Insulin-like growth factor and potassium depolarization maintain neuronal survival by distinct pathways: possible involvement of PI 3-kinase in IGF-1 signaling. J Neurosci 17:1548-1560.

Dohanics J, Hoffman GE, Smith MS, Verbalis JG (1992) Functional neurolobectomy induced by controlled compression of the pituitary stalk. Brain Res 575:215-222.

Dohanics J, Hoffman GE, Verbalis JG (1996) Chronic hyponatremia reduces survival of magnocellular vasopressin and oxytocin neurons after axonal injury. J Neurosci 16:2373-2380.

Franklin JL, Johnson Jr EM (1992) Suppression of programmed neuronal death by sustained elevation of cytoplasmic calcium. Trends Neurosci 15:501-508.

Galli C, Meucci O, Scorziello A, Werge TM, Calissano P, Schettini G (1995) Apoptosis in cerebellar granule cells is blocked by high $\mathrm{KCl}$, forskolin, and IGF-1 through distinct mechanisms of action: the involvement of intracellular calcium and RNA synthesis. J Neurosci 15:1172-1179.

Gallo V, Kingsbury A, Balazs R, Jorgensen OS (1987) The role of depolarization in the survival and differentiation of cerebellar granule cells in culture. J Neurosci 7:2203-2213.

Ghosh A, Carnahan J, Greenberg ME (1994) Requirement for BDNF in activity-dependent survival of cortical neurons. Science 263:1618-1623.

Giovannelli L, Shiromani PJ, Jirikowski GF, Bloom FE (1990) Oxytocin neurons in the rat hypothalamus exhibit c-fos immunoreactivity upon osmotic stress. Brain Res 531:299-303.

Goldberg JL, Barres BA (2000) The relationship between neuronal survival and regeneration. Annu Rev Neurosci 23:579-612.

Hanson Jr MG, Shen S, Wiemelt AP, McMorris FA, Barres BA (1998) Cyclic AMP elevation is sufficient to promote the survival of spinal motor neurons in vitro. J Neurosci 18:7361-7371.

Hare K (1937) Degeneration of the supra-optic nucleus following hypophysectomy in the dog. Am J Physiol 119:326.

Herman JP, Marciano FF, Gash DM (1986) Vasopressin administration prevents functional recovery of the vasopressinergic neurosecretory system following neurohypophysectomy. Neurosci Lett 72:239-246.

Herman JP, Marciano FF, Wiegand SJ, Gash DM (1987) Selective cell death of magnocellular vasopressin neurons in neurohypophysectomized rats following chronic administration of vasopressin. J Neurosci 7:2564-2575.

House SB, Thomas A, Kusano K, Gainer H (1998) Stationary organotypic culture of oxitocin and vasopressin magnocellular neurons from rat and mouse hypothalamus. J Neuroendocrinol 10:849-861.

Huang YS, Dellmann HD (1996) Chronic intermittent salt loading enhances functional recovery from polydipsia and survival of vasopressinergic cells in the hypothalamic supraoptic nucleus following transection of the hypophysial stalk. Brain Res 732:95-105.

Hughes Jr FM, Cidlowski JA (1999) Potassium is a critical regulator of apoptotic enzymes in vitro and in vivo. Adv Enzyme Regul 39:157-171.

Johnson Jr EM, Koike T, Franklin J (1992) A "calcium set-point hypothesis" of neuronal dependence on neurotrophic factor. Exp Neurol 115:163-166.

Kawamoto K, Kawashima S (1985) Plasticity of vasopressin- and oxytocin- 
containing fibers in the ME in hypophysectomized young and old mice. Brain Res 330:189-193.

Koike T, Martin DP, Johnson Jr EM (1989) Role of Ca2+ channels in the ability of membrane depolarization to prevent neuronal death induced by trophic-factor deprivation: evidence that levels of internal $\mathrm{Ca} 2+$ determine nerve growth factor dependence of sympathetic ganglion cells. Proc Natl Acad Sci USA 86:6421-6425.

Ludwig M, Callahan MF, Morris M (1995) Effects of tetrodotoxin on osmotically stimulated central and peripheral vasopressin and oxytocin release. Neuroendocrinology 62:619-627.

Mander TH, Morris JF (1994) Perivascular microglia in the rat neural lobe engulf magnocellular secretory terminals during osmotic stimulation. Neurosci Lett 180:235-238.

Meyer-Franke A, Kaplan MR, Pfrieger FW, Barres BA (1995) Characterization of the signaling interactions that promote the survival and growth of developing retinal ganglion cells in culture. Neuron 15:805-819.

Meyer-Franke A, Wilkinson GA, Kruttgen A, Hu M, Munro E, Hanson Jr MG, Reichardt LF, Barres BA (1998) Depolarization and cAMP elevation rapidly recruit TrkB to the plasma membrane of CNS neurons. Neuron 21:681-693.

Moll JDWD (1962) Observations on the hypothalamo-posthypophyseal system of the posterior lobectomized rat. Gen Comp Endocrinol 2:215-228.

Nakao N (1998) An increase in intracellular levels of cyclic AMP produces trophic effects on striatal neurons developing in culture. Neuroscience 82:1009-1020.

O'Connor W (1952) The normal interphase in polyuria which follows section of the supraoptico-hypophysial tract in the dog. Q J Exp Physiol 37:1-10.

Oppenheim RW (1989) The neurotrophic theory and naturally occurring motoneuron death. Trends Neurosci 12:252-255.

Oppenheim RW (1991) Cell death during development of the nervous system. Annu Rev Neurosci 14:453-501.

Paxinos G, Watson C (1986) The rat brain in stereotaxic coordinates, Ed 2. Orlando, FL: Academic.

Pike CJ, Balazs R, Cotman CW (1996) Attenuation of beta-amyloid neurotoxicity in vitro by potassium-induced depolarization. J Neurochem 67:1774-1777.

Purves D (1988) Body and the brain: a trophic theory of neural connection. Cambridge, MA: Harvard UP.

Raisman G (1973) An ultrastructural study of the effects of hypophysectomy on the supraoptic nucleus of the rat. J Comp Neurol 147:181-207.

Ramon y Cajal S (1928) Degeneration and regeneration of the nervous system. London: Oxford UP.

Robinson AG, Roberts MN, Verbalis JG, Sherman TG (1990) Hyponatremia in rats induces downregulation of vasopressin synthesis. J Clin Invest 86:1023-1029.

Rusnak M, House SB, Arima H, Gainer H (2002) Ciliary neurotrophic factor increases the survival of magnocellular vasopressin and oxytocin neurons in rat supraoptic nucleus in organotypic cultures. Microsc Res Tech 56:101-112.

Rusnak M, House SB, Gainer H (2003) Long-term effects of ciliary neurotrophic factor on the survival of magnocellular vasopresin and oxytocin neurons in supraoptic nucleus in rat hypothalamic slices. J Neuroendocrinol 15:933-999.
Scott DE, Wu W, Slusser J, Depto A, Hansen S (1995) Neural regeneration and neuronal migration following injury. I. The endocrine hypothalamus and neurohypophyseal system. Exp Neurol 131:23-38.

Sharp FR, Sagar SM, Hicks K, Lowenstein D, Hisanaga K (1991) c-fos mRNA, Fos, and Fos-related antigen induction by hypertonic saline and stress. J Neurosci 11:2321-2331.

Shen S, Wiemelt AP, McMorris FA, Barres BA (1999) Retinal ganglion cells lose trophic responsiveness after axotomy. Neuron 23:285-295.

Shiromani PJ, Magner M, Winston S, Charness ME (1995) Time course of phosphorylated CREB and Fos-like immunoreactivity in the hypothalamic supraoptic nucleus after salt loading. Brain Res Mol Brain Res 29:163-171.

Soler RM, Egea J, Mintenig GM, Sanz-Rodriguez C, Iglesias M, Comella JX (1998) Calmodulin is involved in membrane depolarization-mediated survival of motoneurons by phosphatidylinositol-3 kinase- and MAPKindependent pathways. J Neurosci 18:1230-1239.

Svensson M, Aldskogius H (1993) Synaptic density of axotomized hypoglossal motorneurons following pharmacological blockade of the microglial cell proliferation. Exp Neurol 120:123-131.

Tong JX, Vogelbaum MA, Drzymala RE, Rich KM (1997) Radiationinduced apoptosis in dorsal root ganglion neurons. J Neurocytol 26:771-777.

Verbalis JG, Dohanics J (1991) Vasopressin and oxytocin secretion in chronically hyposmolar rats. Am J Physiol 261:1028-1038.

Verbalis JG, Baldwin EF, Robinson AG (1986) Osmotic regulation of plasma vasopressin and oxytocin after sustained hyponatremia. Am J Physiol 250:444-451.

Vutskits L, Bartanusz V, Schulz MF, Kiss JZ (1998) Magnocellular vasopressinergic neurons in explant cultures are rescued from cell death by ciliary neurotrophic factor and leukemia inhibiting factor. Neuroscience 87:571-582.

Watson RE, Wiegand SJ, Clough RW, Hoffman GE (1986) Use of cryoprotectant to maintain long-term peptide immunoreactivity and tissue morphology. Peptides 7:155-159.

Whitnall MH, Key S, Ben-Barak Y, Ozato K, Gainer H (1985) Neurophysin in the hypothalamo-neurohypophysial system. II. Immunocytochemical studies of the ontogeny of oxytocinergic and vasopressinergic neurons. J Neurosci 5:98-109.

Wray S, Kusano K, Gainer H (1991) Maintenance of LHRH and oxytocin neurons in slice explants cultured in serum-free media: effects of tetrodotoxin on gene expression. Neuroendocrinology 54:327-339.

Wu WT, Scott DE, Gilman AM (1989) Correlative scanning-immunoelectromicroscopic analysis of neuropeptide localization and neuronal plasticity in the endocrine hypothalamus. Brain Res Bull 22:399-410.

Yu SP, Yeh CH, Sensi SL, Gwag BJ, Canzoniero LM, Farhangrazi ZS, Ying HS, Tian M, Dugan LL, Choi DW (1997) Mediation of neuronal apoptosis by enhancement of outward potassium current. Science 278:114-117.

Yu SP, Farhangrazi ZS, Ying HS, Yeh CH, Choi DW (1998) Enhancement of outward potassium current may participate in beta-amyloid peptideinduced cortical neuronal death. Neurobiol Dis 5:81-88.

Yu SP, Yeh CH, Gottron F, Wang X, Grabb MC, Choi DW (1999) Role of the outward delayed rectifier $\mathrm{K}+$ current in ceramide-induced caspase activation and apoptosis in cultured cortical neurons. J Neurochem 73:933-941. 